\title{
La minería criminal en el río Atabapo frontera colombo venezolana: propuestas de solución ${ }^{1}$
}

\author{
Mining in criminal Atabapo river venezuelan border colombian: Proposed solutions
}

\author{
Andrea Catalina Fonseca Moreno ${ }^{2}$ \\ Carlos Edgardo Gualteros Pinzón ${ }^{3}$ \\ PhD. Jaime Eduardo González Díaz ${ }^{4}$
}

\section{Resumen}

El propósito de este artículo es describir el fenómeno de la minería criminal en el rio Atabapo límite territorial con Venezuela, y presentar propuestas de solución a este fenómeno. Este es producto de una investigación descriptiva. Presenta un diagnóstico de los aspectos influyentes en el ámbito económico, social, político y militar; y los efectos de estos a futuro de no generarse o contar con una política que permita regular tal actividad. En el aspecto económico los pobladores mayoritariamente indígenas han cambiado la forma de sustentarse debido a la generación de empleo directo por parte de grupos que desarrollan actividades de minería criminal, así también como la afectación de esta actividad en el tema de regalías. En el aspecto social es importante atender la afectación a mediano y largo plazo de la salud de la población de los dos países generada por el vertimiento de elementos como mercurio y cianuro. En el aspecto político, el desconocimiento de la población del área referente a la normatividad minera, la afectación de la soberanía de los dos países dada por la influencia del hombre en el lecho del río. En el ámbito militar actualmente en área y en general en esta parte de la frontera se encuentran grupos al margen de la ley que se ubican estratégicamente en la frontera Colombo-Venezolana con el objetivo de consolidar corredores tácticos de movilidad.

\section{Palabras Claves}

Frontera Colombo-Venezolana, minería criminal, rio Atabapo, ecológico, comunidades

\begin{abstract}
The purpose of this article is to describe the phenomena of criminal mining in the river Atabapo territorial border with Venezuela, and present proposed solutions to this phenomenon. This is a descriptive research product. It presents a diagnosis of influential aspects in the economic, social, political and military fields; and the effects of these futures generated or not to have a policy for regulating such activity. On the economic side the mainly indigenous people have changed the way we sustain due to the generation of direct employment by groups active criminal mining, as well as the involvement of this activity on the issue of royalties. In the social aspect it is important to address the effect on medium and long-term health of the population of the two countries caused by the dumping of elements such as mercury and cyanide. On the political side, ignorance of the area's population concerning mining regulations, the impact on the sovereignty of the two countries given by the influence of man on the riverbed. In the military area currently and generally in this part of the border are groups outside the law are strategically located in the Colombian-Venezuelan border with the aim of consolidating tactical mobility corridors.
\end{abstract}

\section{Keywords}

Colombo-Venezuelan Border, Criminal Mining, Rio Atabapo, Ecological, Communities

Fecha de recepción: 11 de Diciembre de 2018, Fecha de evaluación: 7 de Enero de 2019, Fecha de aceptación: 4 Marzo de 2019

\footnotetext{
1. Artículo derivado del trabajo de grado Prospectiva estratégica del desarrollo de la minería criminal en límite territorial con Venezuela por Andrea Catalina Fonseca Moreno y Carlos Edgardo Gualteros Pinzón, dirigido por Jaime Eduardo González Díaz.

2. Capitán de Corbeta Armada Nacional de Colombia. Profesional en Ciencias Navales. ORCID ID: https://orcid. org/ 0000-00024480-1905

3. Capitán de Corbeta Armada Nacional de Colombia. Profesional en Ciencias Navales

4. Administrador de Empresas. Magister en Desarrollo Empresarial. Doctor en Ciencias Sociales Universidad del Zulia. Profesor asociado Fundación Universitaria Tecnológico Comfenalco Cartagena (Colombia).gonzalezdj@tecnocomfenalco.edu.co. ORCID ID: https://orcid.org/0000-0002-9441-5543.
} 


\section{Introducción}

Aunque algunos hablan de minería ilegal, para efectos de este trabajo se denominara minería criminal por dos razones básicamente, primero, porque las ganancias de esta actividad en parte se destinan al financiamiento de los diferentes grupos al margen de la ley en nuestro país; y segundo, -desde la perspectiva del gobierno nacional- obedeciendo a la necesidad de tener las herramientas jurídicas que permitan la judicialización de aquellos quien en forma no controlada desarrollen esta labor.

Este espacio geográfico está siendo explotado por parte de estos grupos, aprovechando la ausencia del estado, agravando así la situación una población la cual no cuenta con el apoyo del estado para suplir las necesidades básicas como son salud y educación, usurpando la cultura indígena reinante en el área, y explotando en forma indiscriminada no solamente el recurso natural sino a la población misma.

Este análisis se desarrolla no solo tomando en cuenta la afectación a nivel interno de nuestro país, sino que por el contrario las posibles consecuencias de la no atención por parte del estado a nivel externo particularmente con Venezuela quien de una u otra forma se está viendo afectado por la ausencia del estado colombiano en esta zona del país, siendo este un riesgo latente y poco estudiado.

\section{Métodologia}

El presente artículo es producto de una investigación es de carácter descriptivo. Para el desarrollo de la misma se entrevistó personal de la Recoif (Regional de Contrainteligencia Fluvial), de la Armada Nacional de Colombia que se encuentra desarrollando operaciones en el área general del río Atabapo y en sí que tienen contacto cercano con los diferentes líderes tanto gubernamentales, indígenas, como con quienes desarrollan actividad minera en forma ilegal. Igualmente, se utilizaron fuentes secun- darias consistentes en diferentes publicaciones científicas, publicaciones de divulgación, y diferentes textos institucionales. Los resultados fueron procesados por medio del análisis de contenido.

\section{Resultados}

\section{La minería criminal en Colombia}

El concepto de la minería criminal es un concepto nuevo y utilizado en nuestro país. Este concepto o definición se da por parte del gobierno nacional con el ánimo y con el fin de dar a conocer y socializar un problema que afecta diferentes aristas sociales, políticas, económicas, militares y culturales. Este problema se evidencia y se soporta en la explotación de metales y minerales de forma ilegal en sus diferentes formas y métodos de extracción, y no obedeciendo la normatividad existente para dicha actividad, y cuyo fin económico en su gran parte se destina para la financiación de actividades y grupos al margen de la ley.

En el informe presentado por la Presidencia de la República, en temas de seguridad, la minería actualmente está en evolución y se está convirtiendo en una fuente principal de financiación de grupos ilegales, recaudando un aproximado de 7,1 billones de pesos al año, con el agravante de estar presente en más de 233 municipios alrededor del país.

Para entender el papel y la importancia de la minería criminal en el financiamiento de los grupos al margen de la ley, se presenta el siguiente análisis comparativo entre la producción mensual de un kilo de oro en una balsa dedicada a la minería criminal y la producción mensual de un kilo de droga (mariguana o cocaína). Los datos presentados a continuación fueron recolectados en el área por parte de personal de la Regional de Contrainteligencia Fluvial (Recoif) y aunque son públicos, las fuentes utilizadas poseen clasificación reservada. 
Tabla 1: Comparativo entre la minería criminal y el narcotráfico

\begin{tabular}{|c|c|c|c|}
\hline $\begin{array}{c}\text { Producto } \\
(\mathbf{1 K g})\end{array}$ & $\begin{array}{c}\text { Costo de producción } \\
\text { (Aproximado) }\end{array}$ & $\begin{array}{c}\text { Precio de venta } \\
\text { (Aproximado) }\end{array}$ & $\begin{array}{c}\text { Utilidades } \\
\text { (Aproximado) }\end{array}$ \\
\hline Cocaína & $\$ 1.333 .000 .00$ & $\$ 6.466 .365 .00$ & $\$ 5.133 .365 .00$ \\
\hline Pasta de coca & $\$ 727.272 .00$ & $\$ 2.527 .000 .00$ & $\$ 1.799 .728 .00$ \\
\hline Marihuana & $\$ 990.000 .00$ & $\$ 5.000 .000 .00$ & $\$ 4.010 .000 .00$ \\
\hline Oro & $\$ 67.682 .000 .00$ & $\$ 87.480 .000 .00$ & $\$ 29.518 .000 .00$ \\
\hline
\end{tabular}

Fuente: Recoif (2015).

A partir de lo anterior, se puede evidenciar como es que en la actualidad la minería criminal ha desplazado al narcotráfico en los montos de financiamiento en primera medida por las utilidades, y segundo; por la "legalidad" del primero frente al segundo, por ejemplo; la tenencia de narcóticos actualmente es penalizada y sancionada, mientras que la tenencia de elementos tales como el oro no revisten riesgo alguno para los comerciantes ilegales de este elemento.

\section{La minería criminal en el rio Atabapo}

El rio Atabapo Está localizado en el extremo oriental de Guainía; marca el límite entre Colombia y Venezuela. Las poblaciones cercanas son Puerto Inírida y Amanavén, en la desembocadura del Guaviare (Colombia) y San Fernando de Atabapo (Venezuela). Marca el límite entre Colombia y la hermana República de Venezuela en casi todo su recorrido, lleva en su lecho aguas negras brillantes que se convierten en rojas en pequeña cantidad. Es inmensamente ancho, casi dos kilómetros, para su relativamente corta longitud (Figura 1).

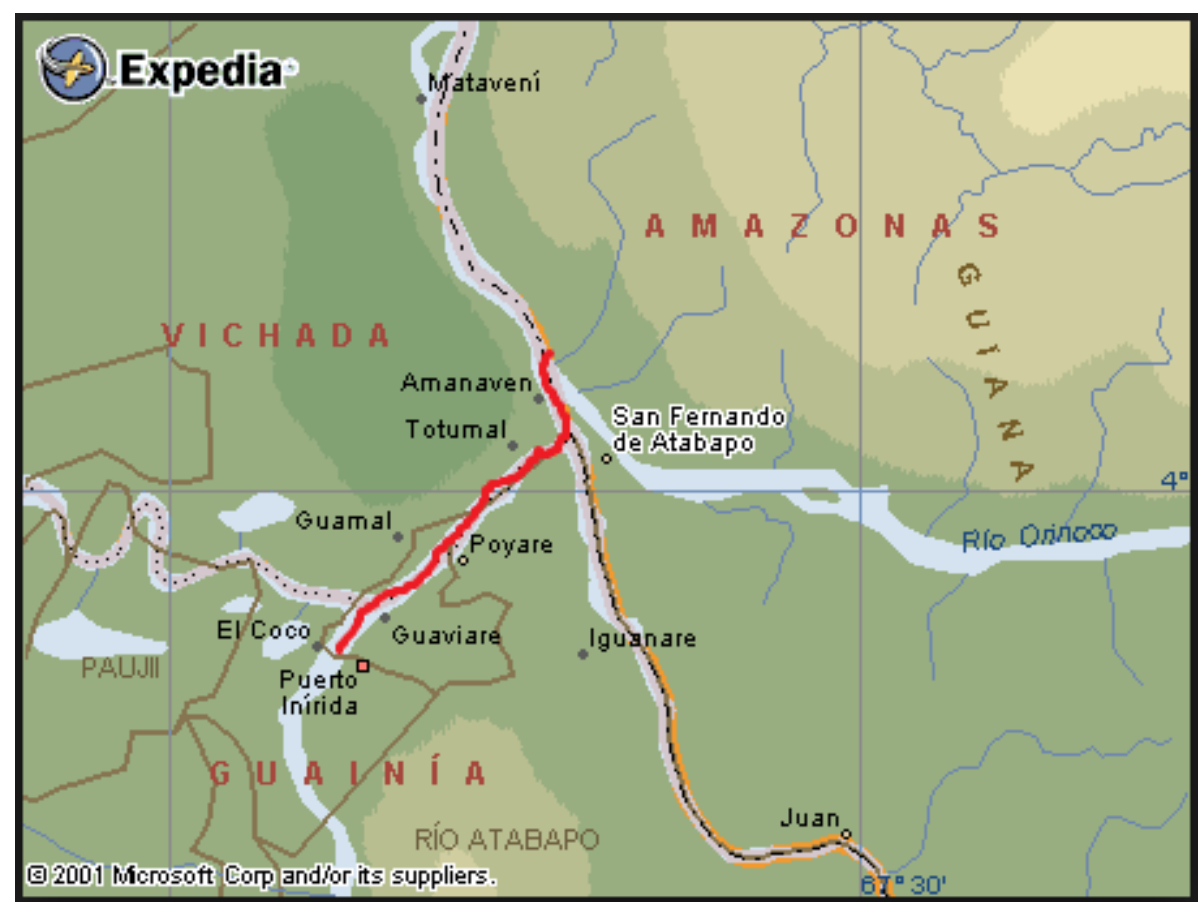

Figura 1. Rio Atabapo

Fuente: Colparques.net

El departamento del Guainía, se caracteriza por poseer una gran riqueza natural, donde el desarrollo y la explotación de la agricultura, pesca y minería se han convertido en las bases o los pilares básicos de la economía de este departamento.
Referente a las vías de comunicación, en este departamento son principalmente fluviales, existen es este departamento dos aeropuertos ubicados en Puerto Inírida y en Barrancominas. Desafortunadamente no existen vías que permitan la comu- 
nicación de este departamento con el centro del país siendo este la principal razón de la ausencia y falta de progreso de la región.

El Atabapo desemboca en el Orinoco y allí forman un accidente hidrográfico sorprendente y único en el planeta: tres ríos confluyen en el mismo punto y dibujan una cruz. El Orinoco forma dos de los parales, el Atabapo uno y el Guaviare el restante. El accidente se observa muy bien desde el aire. En el verano se forman inmensos playones de arena blanca en el río, razón por la cual casi se puede pasar caminando desde Colombia hasta Venezuela (Colparques, 2015).

El río Atabapo está clasificado como río internacional teniendo en cuenta que este si bien no atraviesa si separa y delimita en este caso dos estados. La academia de Derecho Internacional 1956 enuncia que un río internacional "es el que fluye por territorio de dos o más Estados o entre dichos estados. Adicionalmente la convención de 1997 sobre curso de aguas Internacionales, enuncia que “entenderá como un curso de agua Internacional al que alguna de cuyas partes se encuentran en estados distintos" (Gómez, 2007).

Otras condiciones adicionales para que este río sea denominado o clasificado de tipo internacional es que dividen dos estados y adicionalmente sirvan para la navegación. En el caso de no ser navegables se toma la mitad del curso para cada uno de los Estados ribereños. Se deben aplicar los principios de solidaridad y mutua dependencia entre los Estados rivereños, no solo para el río principal si no para la cuenca y sus afluentes (Chavez, 2015).

La minería criminal en Colombia y específicamente en el área en estudio es la denominada minería aluvial. Esta minería es aquella que es desarrollada a las orillas de los ríos. (Min minas y energía, 2003). En el caso particular del río Atabapo, esta funciona de la siguiente manera: los dueños de las plataformas las cuales llamaremos a partir de ahora balsas (Figura 2), las cuales colocan en el borde del río.

Estas balsas están dotadas principalmente por una planta eléctrica de mediano tamaño, dos motobombas y una gran variedad de láminas de colado de diferentes calibres. El proceso desarrollado por estos trabajadores básicamente es la extracción de la arena del río mediante motobombas. Esta mezcla de agua con arena es depositada en las láminas de colado (coladeras) las cuales como su nombre lo indica se encarga de colar o filtrar las partículas sólidas y dejar fluir el agua de regreso al río.

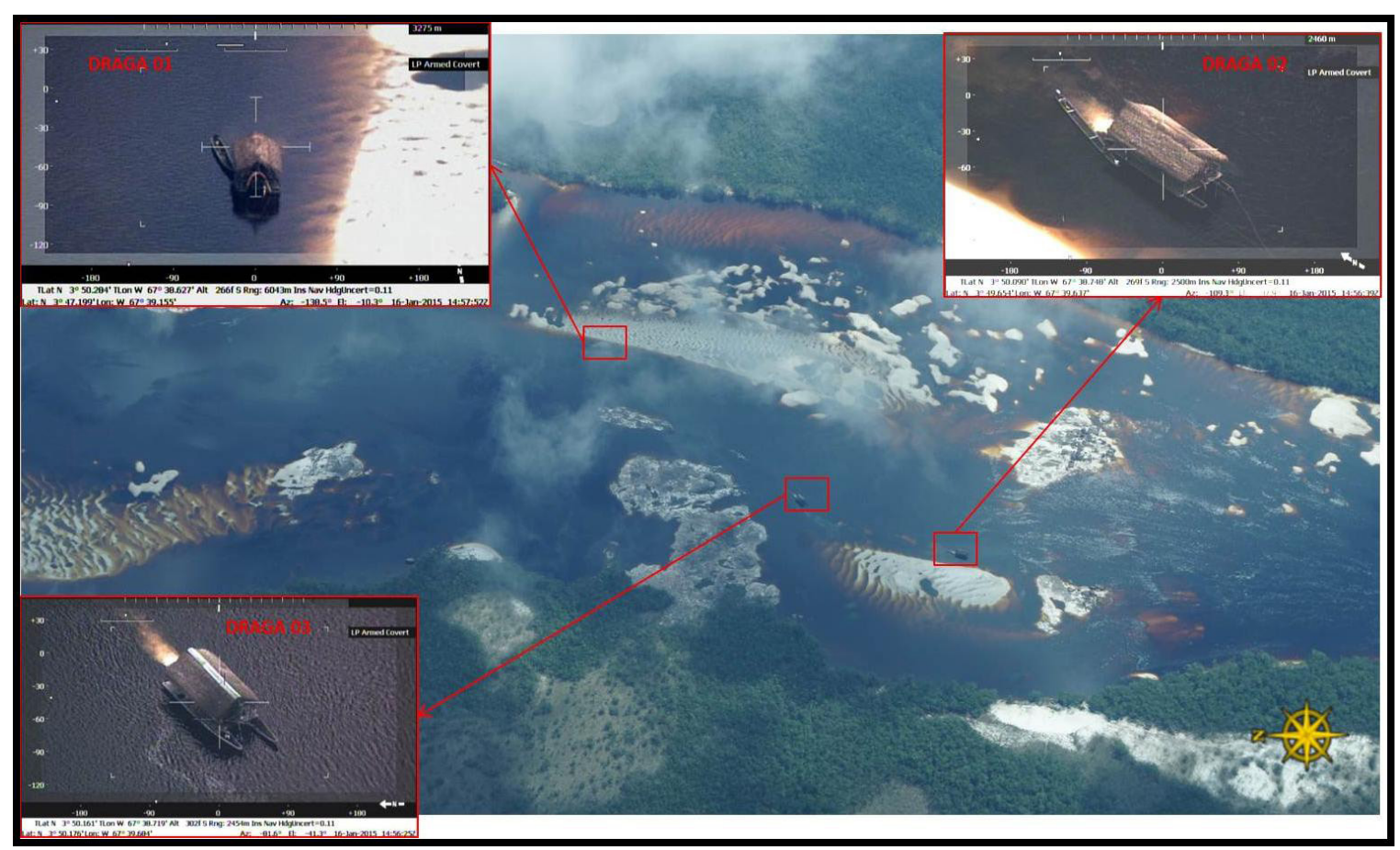

Figura 2. Identificación Balsas Ilegales

Fuente: Regional de Contrainteligencia Fluvial. Armada Nacional de Colombia. 
Posterior a esta labor, esta arena o residuos sólidos es lavada nuevamente con el agua del río, posteriormente utilizan mezclas de cianuro y mercurio con el fin de efectuar el proceso de amalgamación, el cual consiste en la separación de partículas de oro que se encuentran en la arena de tal forma que sean de fácil manipulación. Es de resaltar que esta tarea es la que genera una mayor contaminación de las aguas por vertimiento de estos elementos químicos y tóxicos en estas.

\section{Aspectos influyentes de la minería criminal}

\section{Ámbito económico.}

Tradicionalmente las comunidades del área del río Atabapo basan su economía en la horticultura, la pesca y la cacería. Sin embargo, su principal actividad económica ha sido la elaboración de artesanías tales como canastos, rayadores y objetos de cerámica, y se apoyan con la extracción de pandare, caucho, y fibra de chiquichiqui, que se utiliza para la elaboración de escobas. Igualmente, siguen utilizando en algunos casos el trueque entre los miembros de la comunidad como medio de intercambio para suplir algunas necesidades básicas (Arango y Sánchez, 2004).

Actualmente, han cambiado la forma de sustentarse y obtener beneficios económicos debido a la generación de empleo directo por parte de grupos que desarrollan actividades de minería criminal en el área. Estos grupos utilizan a los indígenas como mano de obra no calificada y para desarrollar labores tales como lavado de arena y buceo artesanal, lo cual genera mayor ganancia y beneficios económicos para estas comunidades, que las actividades derivadas de su economía tradicional (Recoif, 2015).

Es decir, para la operación de las balsas los dueños de las mismas buscan mano de obra barata y no calificada. Son así los indígenas la herramienta más fácil y económica para el desarrollo de estas actividades. Como se señaló arriba, los indígenas son empleados como buzos artesanales los cuales dirigen las mangueras dentro del río de tal forma que se optimice el empleo de las motobombas, y sea entonces la mayor la cantidad posible de arena extraída, colada y procesa- da en búsqueda del oro principalmente. (Recoif, 2015).

Hay que destacar, que hay en la zona personas de estas comunidades indígenas que tienen autorización de explotación de minerales en forma artesanal, estas autorizaciones en muchos de los casos son usadas por estos grupos que extraen recursos en una forma semi industrial e ilegal, los cuales presentan al momento de ser requeridos por las autoridades del área. Esto genera ingresos para muchos indígenas que poseen estos títulos o autorizaciones, en el sentido que por el uso de esos permisos, los mineros ilegales pueden llegar a pagar hasta un millón de pesos mensuales, suma que es elevada para un indígena pero irrisoria para los explotadores de los recursos (Recoif, 2015)

\section{Ámbito social.}

Dentro de las principales afectaciones sociales están los daños a la salud de los pobladores de la región, por un lado, como consecuencia de la utilización de mercurio y cianuro en la producción de oro en las balsas, y por otro lado, y en menor grado por la práctica del buceo no controlado.

La minería ilegal no es nueva en Colombia, en el país se ha estado desarrollando actividades de minería ilegal a lo largo de su historia. Esta actividad ha permitido el desarrollo de investigaciones sobre los efectos de la misma especialmente en la salud del ser humano. Por ejemplo, la Universidad de Antioquia en el año 2009 desarrollo de una investigación denominada "Alteraciones neuropsicológicas en escolares de un municipio con niveles elevados de vapor de mercurio medioambiental", desarrollada en Segovia Antioquia se encontró que el $79.6 \%$ presenta alteraciones en comprensión del lenguaje, el 77.6\% en funciones ejecutivas, el $52,6 \%$ en atención visual, el $43,9 \%$ en fluidez verbal, el $38,8 \%$ en memoria verbal a corto plazo y el $31.1 \%$ en memoria a largo plazo.

Es así importante resaltar que la exposición a vapores de mercurio en forma permanente genera dentro de los menores una afectación en su conducción neural haciendo que se afecte 
su desarrollo intelectual, afectando esto el normal desarrollo del menor, en aspectos como el cognitivo, memoria y lenguaje, factores críticos para el normal desarrollo de un menor y el desempeño del mismo en labores académicas y sociales. Este trabajo enunciado fue realizado tomando una muestra de 196 estudiantes de una población de escolares de 4180, de los cursos de segundo de primaria a noveno de bachillerato (Vargas, B. Quiroz, P. 2009).

En general la exposición a este elemento químico reviste un potencial problema para la salud de las personas. La OMS que si bien todo el mundo está expuesto a este, existen riesgos tales como malformación fetal, desarrollo inadecuado del cerebro y funciones cognitivas, falencias importantes en el desarrollo de la visión, el habla en menores de edad. En los adultos los efectos más notorios, estos se ven afectados con síntomas como cefalea, insomnio, pérdida de la memoria, llegando inclusive a la afectación del sistema urinario causando falla e insuficiencia renal. (OMS, 2013)

El cianuro por su parte es un elemento químico altamente toxico y los efectos para el ser humano pueden llegar a ser letales dependiendo directamente de la concentración del mismo y de los tiempos de exposición. La sintomatología de los efectos a la exposición de estos pueden estar entre un dolor de cabeza, malestar general, irritación en las vías respiratorias, irritación en los ojos, hasta afectación de la tiroides, perdida de la visión, entumecimiento o parálisis muscular y la muerte en muchos de los casos. En el caso de mujeres en estado de embarazo, la exposición a este químico produciría afectación en el desarrollo del feto y en algunos casos se han presentado casos de muerte prenatal (Ramírez, 2010).

Otro de los riesgos a los que se ve expuesta la población del área del río Atabapo y en especial al personal que labora en las balsas son los efectos del buceo no controlado. Sobra decir que el principal riesgo de esto -practicado en su mayoría por indígenas- está en el ahogamiento producido por la falta de experiencia, y sobre todo de controles de seguridad y sanitario que hacen de esta labor una de las que generan mayor riesgo para la comunidad.
Los mayores inconvenientes presentados por parte de estos indígenas, está en enfermedades respiratorias. Se presume que son generadas por la calidad del aire que respiran mientras realizan su labor, lo anterior teniendo en cuenta que los compresores usados no son los indicados para este trabajo, el mantenimiento y limpieza de los mismos no es óptimo y por supuesto el tipo y forma de buceo artesanal el cual no emplea elementos de seguridad tales como reguladores, caretas si no en cambio utilizan mangueras de las cuales toman directamente el aire que respiran. (Recoif, 2015)

Existen una gran variedad de enfermedades tales como enfermedad de descompresión o embolia, narcosis de nitrógeno, entre otras que se relacionan con el buceo en mar, sin embargo el tema de buceo en ríos a profundidades menores a seis metros no está estudiado a fondo por lo que sus efectos en la salud no son científicamente comprobados. (Marqués, R. et al. 2012).

\section{Ámbito político.}

Teniendo en cuenta que la región del rio Atabapo está en zona de frontera con la hermana República de Venezuela, los principales aspectos políticos influyentes en la problemática de la minería criminal estarían relacionados con conflictos fronterizos.

Durante la historia de Colombia y Venezuela, los conflictos limítrofes han ocurrido y han estado desde la misma separación de los países en el año de 1833. Con estos conflictos de intereses tanto terrestres como marítimos, han salido acuerdos y tratados que han permitido definir los límites de los dos países. Tenemos el caso de tratados tales como el laudo español, del 16 de marzo de 1892; el arbitraje suizo, del 24 de marzo de 1892; y el Tratado López de Mesa-Gil Borges, del 5 de abril de 1941. Lo anterior sin mencionar el sinnúmero de acuerdos y pactos de amistad y no agresión entre los dos países en cabeza de los diferentes gobiernos de turno (Buendía, 2016).

En el área del Atabapo entre Colombia y Venezuela existe actualmente vigente el tratado sobre arbitramento "juris" entre los Estados unidos de 
Colombia y los Estados Unidos de Venezuela firmado el 14 de septiembre de 1881. Posterior a las aprobaciones por cada uno de los gobiernos, se tiene que el río Atabapo hace parte de la frontera entre los dos países por un espacio aproximado de $36 \mathrm{~km}$.

Desde el raudal de Maipures por la vaguada del Orinoco hasta su confluencia con el Guaviare, por el curso de este hasta la confluencia del Atabapo, por el Atabapo aguas arriba hasta $36 \mathrm{~km}$ al norte del pueblo de Yavita, trazando una recta que vaya a parar sobre el río Guainía, treinta y seis kilómetros al norte del pueblo de Yabita, trazando desde allí una recta que vaya a parar sobre el río Guainía, treinta y seis kilómetros al occidente del pueblo de Pimichin y por el cauce del Guainía que más adelante toma el nombre de río Negro, hasta la piedra del Cocoy.

Es así como Colombia debe velar por el cumplimiento de estos tratados de tal forma que se garantice la soberanía de la nación sin afectar los intereses de Venezuela. Existe además tratado sobre demarcación de fronteras y navegación de los ríos comunes entre los gobiernos de Colombia y los Estados Unidos de Venezuela, de 1942. Este tratado en su artículo II enuncia la voluntad de los dos países de reconocer recíprocamente y a perpetuidad el derecho de los dos países de efectuar navegaciones en forma libre por los ríos que atraviesen o separen los dos países (ley 3, 1882).

Haciendo énfasis en la necesidad de reglamentar en iguales condiciones aspectos tales como los fiscales, aduaneros y sanitarios en beneficio de la manutención del comercio de los dos países. Sin embargo pone a consideración el derecho de navegación a aquellas embarcaciones que transiten dentro de puertos del mismo país, en donde cada país regulara y controlara de la mejor forma el tránsito de los mismos. (Chávez, 2015)

De otro lado está la convención de Ramsar, este es un acuerdo internacional firmado el 18 de enero del año 1971, y obedece su nombre a la ciudad Iraní donde fue firmada. Esta convención entro en vigor el 21 de diciembre de 1975. Tiene como objetivo fundamental proteger, conservar y hacer uso racional de los humeda- les y las fuentes de agua, recursos hídricos mediante acciones a nivel local, regional y nacional, soportado en la cooperación internacional buscando contribuir al desarrollo sostenible a nivel mundial (Rodríguez, 2004).

Colombia como parte de la convención, tiene inscritos seis sitios Ramsar, los cuales se compromete ante la comunidad internacional de proteger y conservar las características ecológicas de tal forma que sean sostenibles en el tiempo y garanticen de una $\mathrm{u}$ otra forma el abastecimiento de agua y la continuidad en los ecosistemas propios de cada sitio. En el mes de julio del año 2014, Colombia designo el sexto sitio Ramsar de importancia internacional en el territorio nacional. Este sitio está compuesto por el complejo de Humedales de la Estrella Fluvial Inírida ubicada en el Departamento de Guainía (Forero Páez, J. et al. 2016).

Esta zona es conocida como la estrella fluvial de Inirida en el área transicional del Orinoco y el Amazonas y está compuesta por las zonas de confluencia de los ríos Inírida-Guaviare, Guaviare-Atabapo y Guaviare-Ventuari, que se unen al río Orinoco. Esta zona es de importancia ecológica debido a que los bosques inundables pertenecen al bioma amazónico y los diferentes ecosistemas hidrológicos de aguas blancas, negras y transparentes son características del río Orinoco. Esta zona alberga 476 especies de peces $(50 \%$ de las especies para la cuenca del Orinoco y $34 \%$ de las especies del país), 200 especies de mamíferos, 903 de plantas y 324 de aves (Laso, C. et al. 2010).

Durante el proceso de investigación se estableció que los pobladores del lado colombiano se cedulan como venezolanos para hacer uso de beneficios que brinda este país especialmente atención médica y odontológica en Venezuela. Igualmente, acceso a beneficios como mercados y demás ofrecidos por el gobierno para los nacionales venezolanos. Todo esto puede estar relacionado con el descuido del estado colombiano, pero este fenómeno podría ocasionar efectos negativos para la soberanía del país (Recoif, 2015).

Lo anterior cobra importancia frente al tema de fronteras vivas. El fenómeno de fronteras 
vivas, ha sido desarrollado en forma activa en Suramérica por parte de Brasil, quien dentro de sus políticas internas establece que Brasil es estado donde existan nacionales, que no hay límites terrestres sino nacionales haciendo énfasis entre otros aspectos en que en este país no existe variedad ni pluralidad racial, solo existen Brasileros.

Este fenómeno ha sido desarrollado en áreas de la amazonia en donde Brasil ha ganado territorio a lo largo de la historia en sus inicios con la evangelización en zonas selváticas y con poco control estatal y se soporta por la necesidad de este en buscar salida al pacifico al que consideran los brasileros como el futuro del mundo, a tal punto que Brasil ha firmado acuerdos con el gobierno de Ecuador buscando unir la cuenca del río Amazonas con el río Mira y por medio de un puente terrestre lograr la salida al Pacifico sin darle la vuelta al continente por el estrecho de Magallanes o por Panamá. (Chávez, 2015).

Esto es un riesgo latente en la frontera del Atabapo en donde la comunidad claramente está del lado donde obtenga mayores beneficios y en el caso de Colombia se observa un espacio vacío por parte del estado. La presencia de los diferentes entes gubernamentales en el área es limitada y en muchos casos nula. Aspectos tales como salud, educación, carreteras hacen de esta zona del país una zona vulnerable al fenómeno de fronteras humanas.

Parte del riesgo que se evidencia es la posición del estado fronterizo y que se soporta en el artículo 14 de la constitución política venezolana que enuncia "La ley establecerá un régimen jurídico especial para aquellos territorios que por libre determinación de sus habitantes y con aceptación de la Asamblea Nacional, se incorporen al de la República" (Constitución de la República Bolivariana de Venezuela, 1999).

\section{Ámbito militar.}

Actualmente en área del río Atabapo y en general en esta parte de la frontera se encuentran grupos al margen de la ley que se ubican estratégicamente en la frontera Colombo-Venezolana con el objetivo de consolidar corredores tácticos de movilidad los cuales les permiten realizar de forma oportuna la extracción de cocaína medio y método de finanzas y adquisición de materiales de guerra, además del traslado de hombres de la parte Colombiana desde Caño Rayado a Caño Negro frontera con Brasil.

Otro corredor utilizado por estos grupos corresponde a la parte nororiente los municipios de Puerto Inírida (Guainía) y Puerto Carreño (Vichada) por la ladera del río Orinoco y sus corregimientos aledaños, a la parte suroriente desde San Felipe hasta Puerto Colombia por el río Negro y por la parte Venezolana desde el estado de Amazonas poblaciones de Puerto Ayacucho, y las comunidades indígenas que conforman ese estado, entre las cuales una de las más importantes es la comunidad indígena de Yavita lugar donde llegan todas las embarcaciones procedentes de Puerto Ayacucho y de Puerto Inírida, además el área del Estado Bolívar.

Los grupos en mención basan el 70\% de sus finanzas en la minería ilegal (extracción Oro y Tungsteno) mediante el cobro del mal llamado impuesto a la minería, al personal de mineros artesanales de la minas, el objetivo de realizar explotación ellos mismos además por el uso de las pistas, lo cual cobran 30 millones de pesos por vuelo salido (Recoif, 2015).

Otra situación es la afectación de la soberanía y la navegabilidad del rio por cambios en el fondo y variación del curso del mismo por dragado. Durante el proceso de la minería ilegal-criminal, se evidencia la afectación directa del fondo del río por la forma de extracción de la arena como principal insumo dentro de la cadena de explotación del rio. Se estima que por cada balsa instalada se extraen entre $1.5 \mathrm{y}$ dos toneladas de arena, las cuales son tomadas del fondo del río.

Este es un factor que cobra relevancia teniendo en cuenta que el río en la zona de la frontera es navegable, y a largo plazo el desarrollo continuado de esta actividad afectara en forma significativa no solo el lecho del río sino que también será directamente proporcional la afectación de la soberanía de los países limítrofes. (Recoif, 2015) 


\section{Propuestas de solución}

Luego del análisis efectuado se determinaron los siguientes aspectos en los cuales se consi- dera que debe existir una acción inmediata y controlada por parte de los diferentes entes del estado así:

Tabla 2: Aspectos que demandan acción inmediata

\begin{tabular}{|c|c|c|}
\hline Aspecto & Problema & Propuesta de solución \\
\hline Económico & $\begin{array}{l}\text { - Perdida de regalías } \\
\text { - Cambio del sistema económi- } \\
\text { co en el área } \\
\text { - Falta información } \\
\text { - Ausencia de entes de control } \\
\text { fiscal y aduanero }\end{array}$ & $\begin{array}{l}\text { Fortalecer los controles de los recursos } \\
\text { generados por la minería en el Departa- } \\
\text { mento del Guainía. } \\
\text { - Bancarización de la zona. } \\
\text { - Censo de las áreas donde se está desa- } \\
\text { rrollando tanto la minería legal como la } \\
\text { minería ilegal. }\end{array}$ \\
\hline Social & $\begin{array}{l}\text { - Ausencia de hospitales } \\
\text { - Explotación laboral } \\
\text { - Ausencia de escuelas } \\
\text { - Contaminación }\end{array}$ & $\begin{array}{l}\text { - Construir o instalar los centros hospitala- } \\
\text { rios suficientes. } \\
\text { - Plan con el propósito de garantizar en } \\
\text { primera instancia los derechos de los me- } \\
\text { nores. } \\
\text { - Creación de nuevas escuelas. } \\
\text { - Elaboran un plan de control de calidad de } \\
\text { agua. }\end{array}$ \\
\hline Político & $\begin{array}{l}\text { - } \text { Riesgo fronteras vivas. } \\
\text { - } \quad \text { Cambio curso del río. }\end{array}$ & $\begin{array}{l}\text { - Censar, cedular y registrar a todas las co- } \\
\text { munidades en el área del río. } \\
\text { - mantener la soberanía y garantizar la na- } \\
\text { vegación segura. }\end{array}$ \\
\hline Militar & $\begin{array}{l}\text { - Desconocimiento del río en su } \\
\text { cartografía. } \\
\text { - Ausencia de puestos destaca- } \\
\text { dos a lo largo del río } \\
\text { - Ausencia de policía ambiental } \\
\text { y aduanera. }\end{array}$ & $\begin{array}{l}\text { Plan de levantamiento hidrográfico a lo } \\
\text { largo del río Atabapo. } \\
\text { - Instalar los puestos militares a lo largo } \\
\text { del río y de la frontera } \\
\text { - Enviar al área del río Atabapo un grupo } \\
\text { policial de las especialidades medio am- } \\
\text { biente y de aduana. }\end{array}$ \\
\hline
\end{tabular}

Fuente: Elaboración propia

\section{Conclusiones}

En conclusión el país existe la necesidad imperiosa de actualizar y generar normatividad respecto al desarrollo y explotación de la minería. El código minero vigente carece de legislación dada la evolución de medios y métodos de explotación minera en las diferentes zonas del país. Así mismo, esto genera que los diferentes entes del estado encargados de velar por la protección de los recursos no cuenten con las herramientas y los criterios suficientemente bien definidos para desarrollar su trabajo.

El desarrollo de la actividad minera se genera en su gran mayoría en zonas de alta rique- za ecológica donde la presencia del estado es mínima. La afectación a nivel ecológico y ambiental se facilita por la ausencia en general del estado. Estas zonas, incluso muchas de ellas que son de reserva natural requieren atención y presencia debido a que en muchos casos son fuente de suministro hídrico para las diferentes poblaciones cercanas y aledañas a estas.

El área del río Atabapo genera un alto riesgo a nivel geopolítico. Esta zona rica en recursos naturales, con una población predominante indígena, y sirviendo como frontera entre Colombia y Venezuela hacen que se generen problemas 
potenciales de alto impacto para el estado. Lo anterior a que estos recursos están siendo afectados por la mano del hombre, contaminando las fuentes hídricas con el vertimiento de elementos químicos en estos haciendo que se reduzca la producción de pesca en el río, que las poblaciones y grupos indígenas de la zona consuman agua contaminada con elementos tales como cianuro y mercurio los cuales traen consecuencias en la salud a mediano plazo, y consecuencias graves para las próximas generaciones.

La ausencia del estado es aspectos tales como salud y educación, han abierto la oportunidad a que Venezuela desarrolle un proceso de socialización de las diferentes comunidades de la zona del río. Esto se evidencio en la cedulación de estos grupos indígenas y la carnetización de los mismos permitiendo el acceso de estos a la educación y atención medica que el estado Colombiano no les garantiza en el área. Esto eta generando efectos directos en la soberanía bajo el precepto de fronteras vivas, en donde un estado ejerce soberanía no por los límites territoriales sino por los lugares en donde existen nacionales.

Colombia requiere tomar consciencia de la importancia de la minería en el país como fuente de riqueza y de recursos económicos significativos. Aunque se tiene información de un aproximado de las ganancias generadas por los grupos al margen de la ley que dominan la actividad en el área, no se cuentan con las estadísticas confiables que permitan desarrollar ejercicios de planeamiento a nivel estado, no existe un censo en tiempo real de los diferentes sitios de explotación a lo largo y ancho del territorio en parte por la ausencia de entes del estado que efectúen un control de los mismos.

El impacto de la minería en la cultura indígena ha sido alto. Estos grupos han cambiado su forma de vida, sus costumbres y tradiciones para adaptarse a unas que les han sido impuestas por parte de los colonos que han llegado al área a desarrollar la minería. Es así donde se ve la necesidad que el estado intervenga en forma directa con el propósito de garantizar la integridad y continuidad de estos grupos y desarrolle un plan para la explotación responsable de la minería en el área en donde los recursos generados tengan y generen un impacto directo en las comunidades de la zona.

Un punto sensible es la afectación del río por parte de estos grupos ya que la modificación del lecho de estos mediante el movimiento de arena podría generar modificación en el curso del mismo. Es prioridad del estado prestar atención teniendo en cuenta que este río es frontera reconocida y soportada ante la comunidad internacional por los tratados firmados por los dos países, y el cambio del curso del río afectara la soberanía de los dos países.

\section{Referencias bibliográficas}

Arango, R. Sánchez, E. (2004). Los pueblos Indígenas de Colombia en el umbral del nuevo milenio. Departamento Nacional de Planeación, DNP, Dirección de Desarrollo Territorial Sostenible. Bogotá.

Buendía, R. (2009). El problema fronterizo con Venezuela. Periódico el Turbión. Tomado de; http:// elturbion.com/?p=1036. Recuperado 27-10-2015.

Constitución de la República Bolivariana de Venezuela. (1999, 30 de diciembre). Gaceta Oficial de la República, № 36.860.

Contribución a la construcción de un plan estratégico regional para la reducción del uso de mercurio en la minería aurífera artesanal y de pequeña escala. Ministerio del Medio Ambiente. (2012). Tomado de: https:/www.minambiente.gov.co/images/AsuntosambientalesySectorialyUrbana/pdf/ mercurio/Sinopsis_Nacional_de_la_ASGM.pdf. Recuperado el 23 -08-2015. 
Colparques (2015). Rio Atabapo. Tomado de: http://www.colparques.net/ATABAPO. Recuperado 13-08-2015.

Forero Páez, J. C., Granados Acosta, O. L., \& Mora Liscano, W. (2016). La estrella fluvial del Inírida sitio Ramsar como alternativa pedagógica para fortalecer la educación ambiental de la IE custodio García Rovira de Inírida Guainía.

Gómez, J. (2007). Régimen legal de los cursos de aguas internacionales. Afese 42. Tomado de: http:// www.afese.com/img/revistas/revista42/regimen.pdf. Recuperado 13-08-2015.

Gómez, L. (2012). Minería ilegal desde el siglo XVI. contrapunto.com. tomado de: http://contrapunto.co/index.php?module=nota\&i=19-mineria-ilegal-desde-el-siglo. Recuperado 16-08-2015.

Chávez, L. (2015). Minería criminal en el Rio Atabapo. Entrevistado por T. C. Gualteros)

Lasso, C. A., J. S. Usma, F. Trujillo y A. Rial (Editores). 2010. Biodiversidad de la cuenca del Orinoco: bases científicas para la identificación de áreas prioritarias para la conservación y uso sostenible de la biodiversidad. Instituto de Investigación de Recursos Biológicos Alexander von Humboldt, WWF Colombia, Fundación Omacha, Fundación la Salle e Instituto de Estudios de la Orinoquia (Universidad Nacional de Colombia). Bogotá, D. C., Colombia. 609 pp.

Ley 3 de 1882. Tratado sobre arbitramento Juris entre los Estados Unidos de Colombia y los Estados Unidos de Venezuela. Gobierno Colombia y Venezuela. 14 de Septiembre de 1881.

Marqués, R., Kovacs, M., de Lourdes de Azevedo Barbosa, M., Siqueira, G., \& Aguiar, E. (2012). Riesgos percibidos en la práctica del buceo scuba: La perspectiva del consumidor. Estudios y perspectivas en turismo, 21(2), 402-416.

OMS. (2013). Organización Mundial de la Salud. 1 de Septiembre. Tomado de: http://www.who.int/ mediacentre/factsheets/fs361/es/. Recuperado 25-10-2015.

Presidencia de la Republica. (2015). Presidencia de la Republica. Tomado de: http://wp.presidencia.gov.co/Noticias/2015/Julio/Paginas/20150730_02-Gobierno-declara-objetivo-prioritario-de-alto-valor-la-mineria-criminal.aspx. Recuperado 15-10-2015.

Ramírez, A. (2010). Toxicidad del cianuro: investigación bibliográfica de sus efectos en animales y el hombre. Anales de la facultad de medicina, Lima Perú, 63.

Rodríguez, I. (2004). La convención de Ramsar y las aves acuáticas en el neotrópico. Ornitología neotropical, 15, 445-454.

Recoif, f. (15 de Agosto de 2015). Actividades en el rio Atabapo. (T. C. Fonseca, Entrevistador)

Vargas B, M., \& Quiroz P, C. (2009). Alteraciones neuropsicológicas en escolares de un municipio con niveles elevados de vapor de mercurio medioambiental. Facultad nacional de salud pública Uni Antioquia, 461-468. 